\title{
A Common Base Answer to the Question "Which Country is Most Redistributive?"*
}

Peter J. Lambert, University of Oregon, Eugene, OR 97403-1285, USA

plambert@uoregon.edu

Runa Nesbakken, Statistics Norway, NO-0131, Oslo, Norway

runa.nesbakken@ssb.no

Thor O. Thoresen, Statistics Norway, NO-0131, Oslo, Norway

thor.olav.thoresen@ssb.no

\begin{abstract}
We believe that what most authors have in mind when referring to the "most redistributive country" is a tax/transfer schedule that is most redistributive across all pre-tax/transfer income distributions. To measure each country's tax and transfers redistribution according to the same baseline, this paper suggests using the transplant-and-compare method of Dardanoni and Lambert (2002) to establish a common base. The redistributive effects of countries' tax and transfer schedules are illustrated by employing micro data on eight countries from Luxembourg Income Study (LIS). Of the eight, Finland is found to be the most redistributive country according to the common base method.
\end{abstract}

Keywords: Redistributional effect; personal income tax; cross-country comparison; common base JEL Code: H11; H23

\footnotetext{
* This work was supported by the Norwegian Research Council, Grant 187403 . We are grateful to the Luxembourg Income Study (LIS), http://www.lisdatacenter.org for making cross-country income and tax data available, and to Bård Lian and Andrea Papini who have assisted with the work to obtain empirical illustrations. We also thank two anonymous referees for their comments to an earlier version of the paper.
}

\section{Introduction}

Growing concern about the rising income inequality around the world raises the question of how capable the tax-transfer schedule is of reducing inequality. In the literature we find different hypotheses about the relationship between initial income and tax. One of them is the median voter hypothesis (Romer, 1975; Roberts, 1977; Meltzer and Richard, 1981), which suggests that redistribution is increasing in pre-tax income inequality. ${ }^{1}$

To measure the amount of redistribution achieved by the tax-transfer schemes across countries, one often just compares a measure of redistribution for each country; see for example

\footnotetext{
${ }^{1}$ See Milanovic $(2000 ; 2010)$ on the closely related redistribution hypothesis.
} 
OECD $(2008 ; 2011) .^{2}$ But this conventional approach does not necessarily shed light on the question of which country is most redistributive. To answer this question, we would ideally like to determine which country's tax-transfer scheme is most redistributive across all possible pretax distributions. One source of bias associated with the standard approach comes from the fact that the pre-tax income distributions differ across countries. When initial inequality differs across countries, a given tax-transfer system will generate different amounts of redistribution (Musgrave and Thin, 1948). ${ }^{3}$ Another source of bias is the fact that taxes and transfers affect the pre-tax distribution of income, for instance via the effect of income tax on labor supply.

The present paper deals exclusively with the first source of bias. One approach to this problem would be to use microsimulation tools and simulate all the different tax-transfer systems of all countries involved in the comparison. ${ }^{4} \mathrm{~A}$ full comparison along these lines is complicated, however. ${ }^{5}$ Another, and much simpler, approach builds on the transplant-and-compare method of Dardanoni and Lambert (2002). According to this method, a benchmark is established in the form of a common base, against which countries' tax and transfer schedules can be contrasted and compared. This paper uses the transplant-and-compare method to answer the question: which country is most redistributive? The main value added relative to previous work is that we consider taxes and transfers. Previous work using the transplant-and-compare method has only considered tax redistribution (e.g., Lambert et al., 2010; 2012).

The transplant-and-compare method builds on the assumption that the pre-tax income distributions of two countries are isoelastic transformations of one another (see Dardanoni and Lambert, 2002). This means that the distribution of the logarithm of pre-tax income of one country is allowed to differ from the other country only by location and scale. In practice, it is sometimes difficult to characterize the differences between the pre-tax income distributions of several countries only in terms of one parameter for location and another for scale. This restricts the number of countries that can be included in the comparison.

We apply the transplant-and-compare method to data on eight countries in the Luxembourg Income Study Database (LIS, 2010). The LIS database is a high-quality source of internationally comparable microdata on household incomes. Given that we set out to discuss the joint effect of the tax and transfer system, data requirements are extensive, which has also limited

\footnotetext{
${ }^{2}$ For the sake of brevity we will most of the time write, e.g., pre-tax income distributions, rather than pre-tax/transfer income distributions, although we have the entire tax-transfer scheme in mind. Of course, a transfer is a negative tax, so this terminology is also defensible from a conceptual point of view.

${ }^{3}$ Musgrave and Thin (1948, p. 510) write: “. . . the less equal the distribution of income before tax, the more potent will be a (given) progressive tax structure in equalizing income". This issue is also discussed in Bishop et al. (1990) and Seidl et al. (2012).

${ }^{4}$ See Sutherland (2001), for instance.

${ }^{5}$ For one thing, defining tax bases for countries in which a certain source of income is not taxed is very difficult. In their discussion of tax redistribution over time in Norway (1992-2004), Lambert and Thoresen (2009) note that this methodology is difficult to apply even to data on a single country over time.
} 
the number of countries included in the empirical analysis: data on Australia, Denmark, Finland, Greece, Italy, the Netherlands, the United Kingdom, and the United States are used. Of these eight countries, Finland is the most redistributive, while Greece redistributes the least.

The remainder of the paper is organized as follows: Section II provides a brief overview of the literature on redistribution across countries, and Section III presents the measures of redistribution used. The common base method for redistributional comparisons is explained in Section IV. Section V contains our empirical analysis, while Section VI concludes.

\section{Cross-country comparisons}

There is a huge literature comparing the amount of redistribution across countries. Papers in this literature have mainly focused on tax redistribution and most of the time there is no adjustment for the fact that pre-tax income distribution differs across countries.

One example of such a study is the paper by Wagstaff et al. (1999). The paper contains a comprehensive analysis of the redistributional and tax progressivity effects of the personal income tax of twelve OECD countries. They find that there is no link between pre-tax income inequality and the degree of redistribution brought about by personal income tax. Ireland, Spain, the UK and the US are the countries with the most unequal pre-tax income distribution, and they are found to be more redistributive than many other countries, but less redistributive than Sweden and Finland, which have very low levels of pre-tax income inequality. In a similar analysis, Verbist and Figari (2014) find evidence supporting the view that more inequality results in less redistribution.

Bishop et al. (1990), Norregaard (1990), Dardanoni and Lambert (2002) and Seidl et al. (2012) are examples of studies that emphasize the difficulties of comparing tax redistribution when pre-tax distributions differ across countries. As noted by Bishop et al. (1990): "One fiscal system is uniformly more progressive if it is more progressive for all prefisc income distributions" (p. 9). ${ }^{6}$ Bishop et al. (1990) then establish new measures of tax progressivity after controlling for differences in tax and transfer magnitudes between two situations, which are exploited in dominance assessments.

Seidl et al. (2012) also apply a dominance procedure, influenced by the empirical approach presented in Seidl (1994). The starting point is the generalized Lorenz dominance criterion, which applies only to a fixed pre-tax income distribution. In order to approach dominance, they suggest displaying Lorenz curves and concentration curves for income and population quantiles, giving rise to so-called Suits curves. Then pairwise comparisons of

\footnotetext{
6 "Prefisc" means before tax and transfers.
} 
countries are conducted by shifting between tax schedule and income distribution pairs using the same values of population or income quantiles, looking for dominance of tax schedules.

Norregaard (1990) controls for pre-tax income inequality differences by superimposing each country's tax system on a chosen common pre-tax income distribution, with Germany selected as the standard. Dardanoni and Lambert (2002) move further in this direction when applying the transplant-and-compare method to account for the pre-tax differences between countries. Local progression comparisons of Canada, Israel and the UK are carried out to search for unambiguous characterizations in the common base setting. Thus, the present paper adds to the paper by Dardanoni and Lambert by describing how the transplant-and-compare method can be applied in a comparison of global redistribution in a cross-country context. Specifically, we argue that the method can be used to help in finding the most redistributive country.

\section{Measures of redistribution}

Here we briefly introduce the key measures of redistribution that we use. ${ }^{7}$ The ReynoldsSmolensky (1977) index of redistributive effect is the difference in inequality between pre-tax inequality and inequality in disposable income (or net income). If the Gini coefficient is the inequality measure, the Reynolds-Smolensky index equals: $R E^{R S}=G_{X}-G_{Y}$, where $G_{X}\left(G_{Y}\right)$ is the Gini coefficient for pre-tax income (net income). The Pechman-Okner index of redistribution (Pechman and Okner, 1974) is a straightforward alternative, where the absolute difference between pre-tax and net income inequality is normalized by the Gini coefficient for pre-tax income: $R E^{P O}=\left(G_{X}-G_{Y}\right) / G_{X}$. In the following we shall present results for both the ReynoldsSmolensky and the Pechman-Okner indexes. ${ }^{8}$

\section{A common base method}

To establish whether the tax-and-transfer system in country $j$ is more redistributive than the system in country $k$, we would like to apply the two tax systems to the pre-tax distributions of income in both countries. If the tax-and-transfer system of country $j$, say, redistributes more in both countries, then the tax-and-transfer system in $j$ is clearly more redistributive.

\footnotetext{
${ }^{7}$ It is worth noting that choice of index may influence the results of cross-country comparisons. For example, some of the recent evidence, see OECD $(2008 ; 2011)$ and Förster and Whiteford (2009), suggest that the US tax system appears to score well on tax progressivity (compared to other countries), but is lower ranked with respect to tax redistribution (when tax levels are also taken into account).

${ }^{8}$ Luebker (2014) argues in favor of the Pechman-Okner index since it is more consistent with the median voter theorem than the Reynolds-Smolensky index.
} 
However, defining tax-and-transfer schemes for countries where these schemes are not applied is complicated. A much simpler alternative is the common base procedure proposed by Dardanoni and Lambert (2002). The general idea is the following: if pre-tax income distributions are isoelastic transformations of each other, then cross-country redistributional effects can be examined for a common pre-tax income base. The common base pre-tax income distributions are obtained by adjusting their location and scale such that they are comparable with one another. ${ }^{9}$

In practice, the common base method is tantamount to finding $a_{j}$ and $b_{j}$ for each country $j$ such that the difference between the distribution of $a_{j}+b_{j} \ln x_{j}$ (where $x_{j}$ denotes pre-tax income) and a chosen reference distribution is minimized. This is equivalent to estimating the constant $\left(a_{j}\right)$ and the slope $\left(b_{j}\right)$ by OLS in the regression of log pre-tax income in the reference country on log pre-tax income in country $j$. Consequently, the conventional $R^{2}$ can be used as a measure of goodness-of-fit. ${ }^{10}$

In the empirical application we use the pre-tax income distribution of Denmark as the chosen reference distribution. To illustrate the implementation of the common base method further, let us say that we want to adjust the pre-tax income distribution of the United States (US) to that of Denmark (DK). We thus begin by running the regression $\ln x_{i}^{D K}=a_{U S}+b_{U S} \ln x_{i}^{U S}+u_{i}$, where $i$ indexes individuals. ${ }^{11}$ With estimates of $a_{U S}$ and $b_{U S}$, we then proceed to construct the "Denmark-adjusted" net income in the US: $y_{i}^{U S^{g}}=\exp \left\{\hat{a}_{U S}+\hat{b}_{U S} \operatorname{lny}{ }_{i}^{U S}+\hat{u}_{i}\right\}$, where the superscript $g$ signifies that US incomes are reshaped by the difference in pre-tax income inequality between Denmark and the US, and $\hat{u}_{i}$ denotes a random draw from the residual distribution. Denote the Gini of the Denmark-adjusted distribution of net income by $G_{Y}^{U S^{g}}$. Proceeding in an analogous fashion, we can construct the Gini of the Denmark-adjusted distribution of the pre-tax income distribution, $G_{X}^{U S^{8}}$. Now, a common base comparison of the Reynolds-Smolensky redistribution between Denmark and the

\footnotetext{
${ }^{9}$ We refer the reader to Dardanoni and Lambert (2002) for a formal presentation of the method. Broadly, the idea is as follows. Consider two countries in which the distributions of the logarithm of pre-tax incomes differ only by location and scale. Then it is possible to find an isoelastic "deformation function" that adjusts for the difference in the pre-tax distribution of income in the two countries.

${ }^{10}$ A two-parameter model of income distribution may clearly be restrictive; for example, the upper part of the distribution may not be well described. However, as discussed in detail in Dardanoni and Lambert (2002), the method rests on the isoelastic form. Here we reshape by using the lognormal distribution. Other suitable families for such standardizations are the Pareto and the SinghMaddala.

${ }^{11}$ A small complication is that the number of observations per country has to be the same. The LIS data contain 84,673 observations for Denmark and 66,832 for the US. We convert these to a fixed sample size of 10,000 by averaging. Alternative methodologies, e.g., random draws, for reducing the data to a given sample size across countries do not change the results.
} 
US can be carried out by comparing the redistribution in Denmark, as obtained from the data, to the reshaped redistribution index for the US, $G_{X}^{U S^{g}}-G_{Y}^{U S^{g}}$. In practice one may use the actual Gini for the pre-tax distribution in Denmark, $G_{X}^{D K}$ in place of $G_{X}^{U S^{8}}$ since $G_{X}^{D K}$ is the base that $G_{X}^{U S^{g}}$ is intended to replicate. ${ }^{12}$ This is also what we do in the empirical analysis.

Although convenient to implement, there are a couple of caveats associated with our empirical approach. One obvious caveat is related to methodology. As already mentioned, we must be able to describe one distribution as an isoelastic transformation of another distribution. If the isoelastic transformation is not approximately valid, the transplant-and-compare methodology may yield misleading results. Another important caveat is that our empirical analysis does not take account of behavioral responses to the tax-and-transfer system. ${ }^{13}$

\section{Which country is most redistributive?}

\section{Selection of countries}

We implement the transplant-and-compare method by providing estimates of tax/transfer redistribution for a selection of countries in the LIS database: Australia (AU), Denmark (DK), Finland (FI), Greece (GR), Italy (IT), the Netherlands (NL), the United Kingdom (UK) and the United States (US).

Data availability is a constraint in our study. As a measure of pre-tax income, we use factor P income (market income), consisting of factor income, private transfers and public pensions; see Milanovic (2000). Social security transfers are excluded, except for pensions, which can be seen as deferred wage payments. In our case, factor $P$ income includes old age universal pensions, disability universal pensions, and survivors' universal pensions. Importantly, social insurance transfers, social assistance transfers and some universal transfers (for instance, unemployment benefits, family allowances and social assistance) are omitted from this income concept. Due to missing information in LIS, we have only been able to establish this income concept for a relatively small number of countries. ${ }^{14}$

Moreover, we are forced to exclude some countries, since we are unable to find a reliable isoelastic transformation. For some countries, the $R^{2}$ is low, suggesting that the two-parameter

\footnotetext{
${ }^{12}$ For further discussion, see Appendix C in Lambert and Thoresen (2009) (although this paper considers intertemporal comparison of tax schedules).

${ }^{13}$ See Thoresen et al. (2012) for a discussion of how behavioral effects can be accounted for in a common base procedure (although exemplified with discussion of tax redistribution over time in one country).

${ }^{14}$ When a broader income concept that includes long-term insurance and assistance benefits was used, data on more countries were available. However, we found it difficult to establish a common base for that income concept. It is also worth noting that experience from studies of common base tax redistribution, as in Lambert et al. (2010), demonstrates that the method works well for a large number of countries when it is confined to analyzing tax redistribution.
} 
representation is too restrictive. As a result, it is difficult to replicate the income distribution of the base country, i.e., Denmark, with data from the country in question. ${ }^{15}$ The countries listed above are the ones for which we have sufficient data and where the isoelastic transformation works relatively well. We thus analyze the question - "which country is most redistributive?" - solely within this particular selection of countries. With that said, we also note that very different welfare regimes of the industrialized world are represented within this set of countries. Our analysis includes the Anglo-Saxon countries (Australia, the UK, and the US), the Nordic countries (Denmark and Finland), Central Europe (the Netherlands), and Southern Europe (Greece and Italy).

\section{Contrasting common base redistribution with the results of the standard approach}

In this section we first report the results of the conventional "between country approach" and then we compare these results with redistribution according to the common base methodology.

Figure 1 shows the relationship between the Reynolds-Smolensky (left-hand panel) and Pechman-Okner (right-hand panel) indexes of redistribution and pre-tax inequality across countries. Note that the estimates of pre-tax inequality in the respective countries are close to the inequality in market income reported in OECD (2011) for the time period 2006-2009.

\section{Figure 1. Results of standard approach to measuring the relationship between redistribution and pre-tax income inequality}


Notes: LIS data, 2010 (Wave VIII). The slope of the regression line is 0.33 (standard error: 0.31) in the left-hand panel and 0.05 (standard error: 0.72 ) in the right-hand panel.

On average there is a positive relationship between pre-tax inequality and redistribution. But the relationship is weak, as indicated by the fact that the slopes of the

\footnotetext{
${ }^{15}$ Note that using another common base in place of Denmark does not solve this problem.
} 
regression lines are not statistically significant. ${ }^{16} \mathrm{We}$ also note that Finland is the most redistributive country according to both indexes.

Figure 1 also shows that the index of redistribution that is chosen matters for the results. The Reynolds-Smolensky index suggests that there is a lot of redistribution in the UK. However, this result seems to be partly driven by the fact that there is considerable pre-tax inequality in the UK. When the differences in the Gini coefficients are normalized with initial inequality, there is less redistribution in the UK than in the Netherlands, Italy, and Denmark; see right-hand panel.

Let us now turn to the common base results. In order to contrast these results with the results obtained when using standard techniques (Figure 1), we employ the same redistribution indexes for the common base results as are used in the standard depiction. ${ }^{17}$

An important step in the common base analysis is to estimate the transformations required to make pre-tax income distributions across countries comparable. Appendix Table A1 presents these OLS regression results. In addition, the table reports the deviations between the Gini coefficients of the adjusted (transplanted) pre-tax income distributions and the income distribution of Denmark, to which they are fitted. The table reveals that most transplants are close to the target. However, for Greece, inequality according to the adjusted distribution exceeds inequality in Denmark by more than 20 percent. This may suggest that some care should be exercised when interpreting the results for Greece. ${ }^{18}$

\section{Figure 2. Results of common base approach to measuring the relationship between redistribution and pre-tax income inequality}
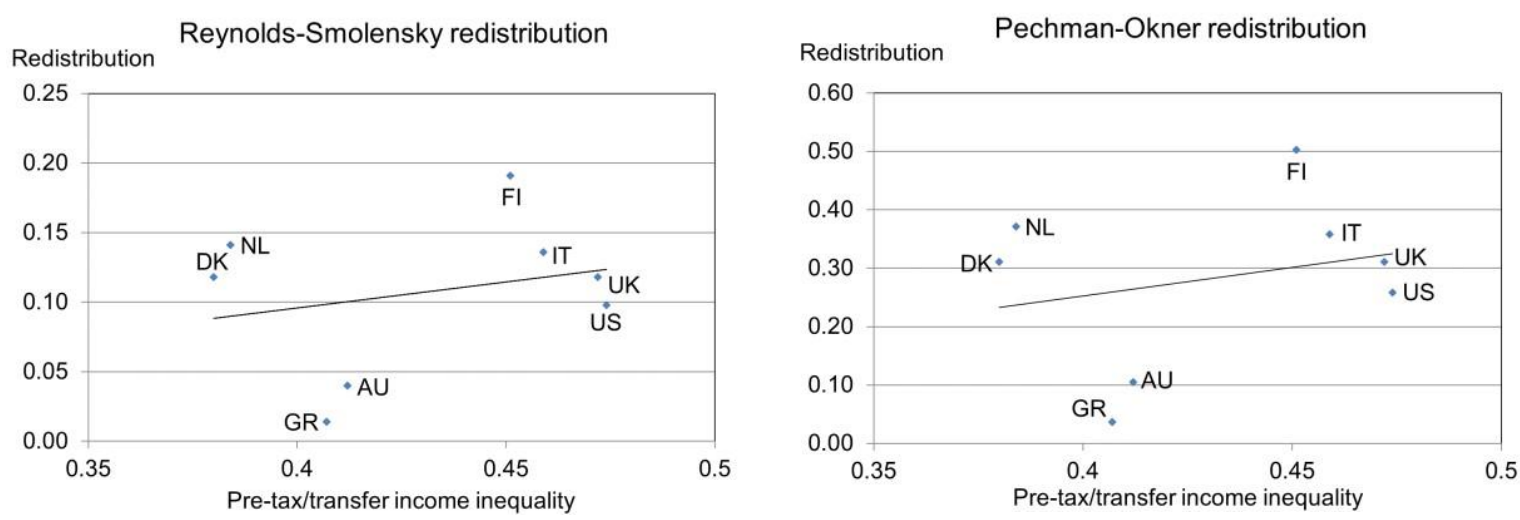

Notes: LIS data, 2010 (Wave VIII). The slope of the regression line is 0.33 (standard error: 0.59) in the left-hand panel and 0.86 (standard error: 1.55) in the right-hand panel.

\footnotetext{
${ }^{16}$ Interpreted literally, there is thus only weak support for the median voter hypothesis.

17 This is not necessary since, by construction, $G_{X}$ is held constant across countries. It would thus be sufficient to compare $G_{Y}$ across countries, where a lower Gini indicates more redistribution.

${ }^{18}$ Another vital precursor to the analysis is to verify that the choice of reference distribution does not influence the results. Appendix Figure A1 shows redistribution for a selection of five countries, using the pre-tax income distribution of each of those five countries in turn as reference. In general, the choice of base is not important for the results.
} 
Figure 2 presents the results from the common base analysis. Again, Finland is the most redistributive country, while there is least redistribution in Greece. However, this does not necessarily imply that choice of method is unimportant. To illustrate this, Figure 3 compares the results from the standard method (horizontal axes) and the common base method (vertical axes) for the two indexes of redistribution. The left-hand panel pertains to the Reynolds-Smolensky index, while the right-hand panel shows the results for the Pechman-Okner index. The numbers within parentheses report the country rank associated with the standard method (the first position) and the common base method (the second position).

Figure 3 shows that, with the exception of Greece and Australia, the common base adjustment implies relatively small changes in the redistribution index. But even small changes in measured redistribution can affect the country rankings. The biggest change in the country ranking is for the UK in the case of Reynolds-Smolensky redistribution. The standard method suggests that there is substantial redistribution in the UK, and the UK would thus be ranked second according to this method. But since pre-tax inequality is high in the UK, the UK tax-and-transfer system appears to be more redistributive than it really is. Accordingly, the common base approach, which adjusts for differences in pre-tax inequality, implies that the UK drops to rank 5.

\section{Figure 3. Standard approach and common base redistribution compared}
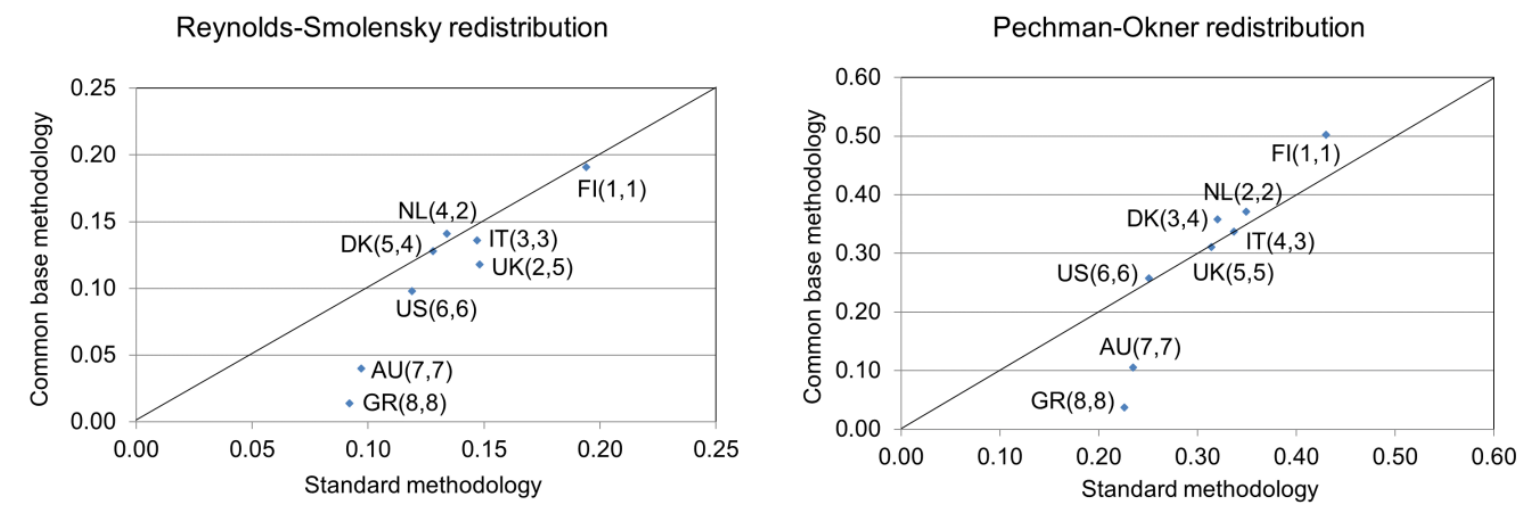

Notes: LIS data, 2010 (Wave VIII). The first number within parentheses report the country rank according to the standard method, while the second number reports the country rank according to the common base method. The straight lines are 45-degree lines.

\section{Conclusion}

When discussing how redistributive tax-and-transfer systems are across the world, we would ideally like to hold pre-tax income inequality constant. This is not how typical cross-country comparisons are conducted, however. More often than not, there is no adjustment for pre-tax inequality, which is then a confounding factor in comparative work.

We have used the transplant-and-compare method of Dardanoni and Lambert (2002) to adjust for differences in pre-tax inequality across countries. Applying this (common base) 
method, we find that the tax-and-transfer system in Finland is the most redistributive among the eight countries - Australia, Denmark, Finland, Greece, Italy, the Netherlands, the United Kingdom, and the United States - considered in this study.

We see the transplant-and-compare method as a move in the right direction, improving the level of comparability in cross-country studies. However, there are some limitations to the approach. Most importantly, it relies on the assumption that the pre-tax distribution of income in one country is an isoelastic transformation of the pre-tax distribution in another country. This assumption implies that two parameters, location and scale, are sufficient to capture the difference between distributions in any two countries. This representation does not work well for all bilateral comparisons, a problem that has reduced the number of countries included in the empirical application.

In the standard (naive) cross-country comparison, the choice of redistribution index (Reynolds-Smolensky or Pechman-Okner) affects the results. This is because these indexes adjust for pre-tax inequality in different ways. By contrast, the common base method we have considered holds pre-tax inequality constant and therefore country rankings do not vary with the type of redistribution index. We think this is an important advantage of the transplant-andcompare method.

\section{References}

Bishop J.A., Formby, J.P., and Thistle, P.D. (1990), International Comparisons of Tax and Transfer Progressivity: New Evidence from the Luxembourg Income Study, Working Paper No. 52, Luxembourg Income Study.

Dardanoni, V. and Lambert, P.J. (2002), Progressivity Comparisons, Journal of Public Economics $86,99-122$.

Förster, M. and Whiteford, P. (2009), How much Redistribution do Welfare States Achieve? The Role of Cash Transfers and Household Taxes, CESifo DICE Report 3/2009.

Lambert, P.J., Nesbakken, R., and Thoresen, T.O. (2010), On the Meaning and Measurement of Redistribution in Cross-Country Comparisons, LIS Working Paper Series, No. 532, Luxembourg Income Studies.

Lambert, P.J., Nesbakken, R., and Thoresen, T.O. (2012), Is There More Redistribution in Scandinavia than in the US? Economics Bulletin 32, 2146-2154.

Lambert, P.J. and Thoresen, T.O. (2009), Base Independence in the Analysis of Tax Policy Effects: With an Application to Norway 1992-2004, International Tax and Public Finance 16, 219-252. 
Luebker, M. (2014), Income Inequality, Redistribution, and Poverty: Contrasting Rational Choice and Behavioral Perspectives, Review of Income and Wealth 60, 133-154.

Luxembourg Income Study (LIS, 2010), Luxembourg Income Study Database, multiple countries in Wave VIII (2010), Luxembourg: LIS. http://www.lisdatacenter.org.

Meltzer, A.H. and Richard, R.F. (1981), A Rational Theory of the Size of Government, Journal of Political Economy 89, 914-27.

Milanovic, B. (2000), The Median-Voter Hypothesis, Income Inequality, and Income Redistribution: An Empirical Test with the Required Data, European Journal of Political Economy 16, 367-410.

Milanovic, B. (2010), Four Critiques of the Redistribution Hypothesis: An Assessment. European Journal of Political Economy 26, 147-154.

Musgrave, R.A. and Thin, T. (1948), Income Tax Progression, 1929-1948, Journal of Political Economy 56, 498-514.

Norregaard, J. (1990), Progressivity of Income Tax Systems, OECD Economic Studies 15, 83110.

OECD (2008), Growing Unequal? Income Distribution and Poverty in OECD Countries, OECD Publishing, Paris.

OECD (2011), Divided We Stand: Why Inequality Keeps Rising, OECD Publishing, Paris.

Pechman, J.A. and Okner, B. (1974), Who Bears the Tax Burden? Brookings Institution, Washington D.C.

Reynolds, M. and Smolensky, E. (1977), Public Expenditures, Taxes, and the Distribution of Income: The United States, 1950, 1961, 1970, Academic Press, New York.

Roberts, K.W.S. (1977), Voting Over Income Tax Schedules, Journal of Public Economics 8, 329-40.

Romer, T. (1975), Individual Welfare, Majority Voting, and the Properties of the Linear Income Tax, Journal of Public Economics 4, 163-185.

Seidl, C. (1994), Measurement of Tax Progression with Nonconstant Income Distributions, in W. Eichhorn (ed.), Models and Measurement of Welfare and Inequality, Springer-Verlag, Berlin.

Seidl, C., Pogorelskiy, K., and Traub, S. (2012), Tax Progression in OECD Countries. An Integrative Analysis of Tax Schedules and Income Distributions, Springer, Heidelberg, New York.

Sutherland, H. (2001), Final Report EUROMOD: An Integrated European Benefit-Tax Model, EUROMOD Working Paper No. EM9/01.

Thoresen, T.O., Bø, E.E., Fjærli, E., and Halvorsen, E. (2012), A Suggestion for Evaluating the Redistributional Effects of Tax Changes: With an Application to the 2006 Norwegian Tax Reform, Public Finance Review 40, 303-338. 
Thoresen, T.O., Jia, Z., and Lambert, P.J. (2016), Is there More Redistribution Now? A Review of Methods for Evaluating Tax Redistributional Effects, FinanzArchiv/Public Finance Analysis 72, 302-333.

Verbist, G. and Figari, F. (2014), The Redistributive Effect and Progressivity of Taxes Revisited: An International Comparison across the European Union, FinanzArchiv/Public Finance Analysis 70, 405-429.

Wagstaff, A., et al. (1999), Redistributive Effect, Progressivity and Differential Tax Treatment: Personal Income Taxes in Twelve OECD Countries, Journal of Public Economics 72, 73-98.

\section{Appendix}

Figure A1. Common base redistribution (Reynolds-Smolensky) in five countries, with different base countries

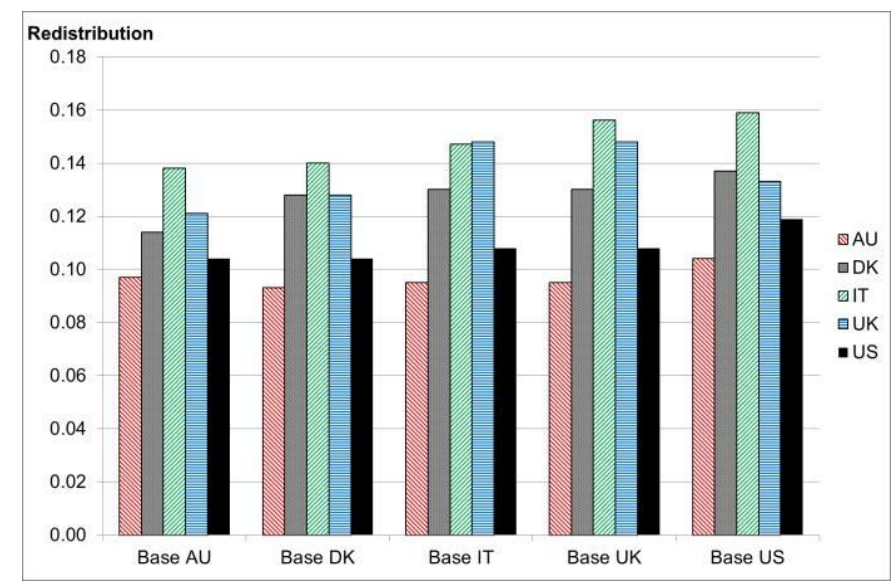

Table A1. Results of OLS regressions with log of pre-tax/transfer income of Denmark as the dependent variable

\begin{tabular}{lcccc}
\hline Country & Intercept, $a$ & Slope, $b$ & $R^{2}$ & $\begin{array}{c}\text { Pre-tax/transfer } \\
\text { income inequality } \\
\text { (Gini) of transplants }\end{array}$ \\
\hline Australia (AU) & $1.629(0.022)$ & $1.014(0.002)$ & 0.959 & $0.433(13.9 \%)$ \\
Finland (FI) & $6.259(0.014)$ & $0.633(0.001)$ & 0.976 & $0.346(-8.9 \%)$ \\
Greece (GR) & $2.001(0.033)$ & $1.100(0.004)$ & 0.954 & $0.458(20.5 \%)$ \\
Italy (IT) & $5.831(0.014)$ & $0.702(0.002)$ & 0.977 & $0.384(1.1 \%)$ \\
Netherlands (NL) & $3.777(0.016)$ & $0.853(0.002)$ & 0.966 & $0.355(-6.6 \%)$ \\
UK & $5.753(0.015)$ & $0.695(0.002)$ & 0.951 & $0.390(2.6 \%)$ \\
US & $4.844(0.012)$ & $0.740(0.001)$ & 0.977 & $0.386(1.6 \%)$ \\
\hline
\end{tabular}

Notes. LIS data, 2010 (Wave VIII). Standard errors in parentheses in the reporting of estimates of intercept and slope.

a. Relative difference from actual value for Denmark (0.380) in parentheses. 